\title{
The generalized Weyl Poisson algebras and their Poisson simplicity criterion
}

\author{
V. V. Bavula ${ }^{1}$
}

Received: 2 February 2019 / Revised: 12 July 2019 / Accepted: 4 September 2019 /

Published online: 27 September 2019

(c) The Author(s) 2019

\begin{abstract}
A new large class of Poisson algebras, the class of generalized Weyl Poisson algebras, is introduced. It can be seen as Poisson algebra analogue of generalized Weyl algebras or as giving a Poisson structure to (certain) generalized Weyl algebras. A Poisson simplicity criterion is given for generalized Weyl Poisson algebras, and an explicit description of the Poisson centre is obtained. Many examples are considered (e.g. the classical polynomial Poisson algebra in $2 \mathrm{n}$ variables is a generalized Weyl Poisson algebra).
\end{abstract}

Keywords A generalized Weyl Poisson algebra · A Poisson algebra $\cdot$ The Poisson centre $\cdot$ A Poisson prime ideal · The Poisson simplicity

Mathematics Subject Classification 17B63 $\cdot$ 17B65 $\cdot$ 17B20

\section{Contents}

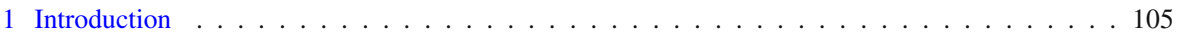

2 The generalized Weyl Poisson algebras . . . . . . . . . . . . . . . . . . . . 108

3 Poisson simplicity criterion for generalized Weyl Poisson algebras . . . . . . . . . . . . . 115

References . . . . . . . . . . . . . . . . . . . . . . . . . . . 119

\section{Introduction}

In this paper, $K$ is a field, algebra means a $K$-algebra (if it is not stated otherwise) and $K^{*}=K \backslash\{0\}$.

Generalized Weyl algebras, [1-3]. Let $D$ be a ring, $\sigma=\left(\sigma_{1}, \ldots, \sigma_{n}\right)$ be an $n$-tuple of commuting automorphisms of $D, a=\left(a_{1}, \ldots, a_{n}\right)$ be an $n$-tuple of elements of

$\bowtie$ V. V. Bavula

v.bavula@sheffield.ac.uk

1 Department of Pure Mathematics, University of Sheffield, Hicks Building, Sheffield S3 7RH, UK 
the centre $Z(D)$ of $D$ such that $\sigma_{i}\left(a_{j}\right)=a_{j}$ for all $i \neq j$. The generalized Weyl algebra $A=D[X, Y ; \sigma, a]$ (briefly GWA) of rank $n$ is a ring generated by $D$ and $2 n$ indeterminates $X_{1}, \ldots, X_{n}, Y_{1}, \ldots, Y_{n}$ subject to the defining relations:

$$
\begin{aligned}
Y_{i} X_{i} & =a_{i}, \quad X_{i} Y_{i}=\sigma_{i}\left(a_{i}\right), \quad X_{i} d=\sigma_{i}(d) X_{i}, \quad Y_{i} d=\sigma_{i}^{-1}(d) Y_{i} \quad(d \in D), \\
{\left[X_{i}, X_{j}\right] } & =\left[X_{i}, Y_{j}\right]=\left[Y_{i}, Y_{j}\right]=0, \quad \text { for all } i \neq j,
\end{aligned}
$$

where $[x, y]=x y-y x$. We say that $a$ and $\sigma$ are the sets of defining elements and automorphisms of the GWA $A$, respectively.

The $n$ 'th Weyl algebra $A_{n}=A_{n}(K)$ over a field (a ring) $K$ is an associative $K$ algebra generated by $2 n$ elements $X_{1}, \ldots, X_{n}, Y_{1}, \ldots, Y_{n}$, subject to the relations:

$$
\left[Y_{i}, X_{i}\right]=\delta_{i j} \text { and }\left[X_{i}, X_{j}\right]=\left[Y_{i}, Y_{j}\right]=0 \text { for all } i, j
$$

where $\delta_{i j}$ is the Kronecker delta function. The Weyl algebra $A_{n}$ is a generalized Weyl algebra $A=D[X, Y ; \sigma ; a]$ of rank $n$ where $D=K\left[H_{1}, \ldots, H_{n}\right]$ is a polynomial ring in $n$ variables with coefficients in $K, \sigma=\left(\sigma_{1}, \ldots, \sigma_{n}\right)$ where $\sigma_{i}\left(H_{j}\right)=H_{j}-\delta_{i j}$ and $a=\left(H_{1}, \ldots, H_{n}\right)$. The map

$$
A_{n} \rightarrow A, \quad X_{i} \mapsto X_{i}, \quad Y_{i} \mapsto Y_{i}, \quad i=1, \ldots, n,
$$

is an algebra isomorphism (notice that $Y_{i} X_{i} \mapsto H_{i}$ ).

It is an experimental fact that many quantum algebras of small Gelfand-Kirillov dimension are GWAs (e.g. $U\left(\mathrm{sl}_{2}\right), U_{q}\left(\mathrm{sl}_{2}\right)$, the quantum Weyl algebra, the quantum plane, the Heisenberg algebra and its quantum analogues, the quantum sphere and many others).

The GWA-construction turns out to be a useful one. Using it for large classes of algebras (including the mentioned ones above), all the simple modules were classified, explicit formulae were found for the global and Krull dimensions, their elements were classified in the sense of Dixmier [5], etc.

The generalized Weyl Poisson algebra $D[X, Y ; a, \partial\}$. Our aim is to introduce a Poisson algebra analogue of generalized Weyl algebras. An associative commutative algebra $A$ is called a Poisson algebra if it is a Lie algebra $(A,\{\cdot, \cdot\})$ such that $\{a, x y\}=$ $\{a, x\} y+x\{a, y\}$ for all elements $a, x, y \in D$. Let $A$ be a Poisson algebra with Poisson bracket $\{\cdot, \cdot\}, \operatorname{PZ}(A):=\{a \in A \mid\{a, x\}=0$ for all $x \in A\}$ be its Poisson centre and $\operatorname{PDer}_{K}(A)$ be the set of derivations of the Poisson algebra $A$ (see Sect. 2 for details).

Definition Let $D$ be a Poisson algebra, $\partial=\left(\partial_{1}, \ldots, \partial_{n}\right) \in \operatorname{PDer}_{K}(D)^{n}$ be an $n$-tuple of commuting derivations of the Poisson algebra $D, a=\left(a_{1}, \ldots, a_{n}\right) \in \mathrm{PZ}(D)^{n}$ be such that $\partial_{i}\left(a_{j}\right)=0$ for all $i \neq j$. The generalized Weyl algebra

$$
\begin{aligned}
A & =D\left[X, Y ;\left(\operatorname{id}_{D}, \ldots, \operatorname{id}_{D}\right), a\right] \\
& =D\left[X_{1}, \ldots, X_{n}, Y_{1}, \ldots, Y_{n}\right] /\left(X_{1} Y_{1}-a_{1}, \ldots, X_{n} Y_{n}-a_{n}\right)
\end{aligned}
$$

admits a Poisson structure which is an extension of the Poisson structure on $D$ and is given by the rule: For all $i, j=1, \ldots, n$ and $d \in D$, 


$$
\begin{aligned}
\left\{Y_{i}, d\right\} & =\partial_{i}(d) Y_{i}, \quad\left\{X_{i}, d\right\}=-\partial_{i}(d) X_{i} \text { and }\left\{Y_{i}, X_{i}\right\}=\partial_{i}\left(a_{i}\right), \\
\left\{X_{i}, X_{j}\right\} & =\left\{X_{i}, Y_{j}\right\}=\left\{Y_{i}, Y_{j}\right\}=0 \text { for all } i \neq j .
\end{aligned}
$$

The Poisson algebra is denoted by $A=D[X, Y ; a, \partial\}$ and is called the generalized Weyl Poisson algebra of rank $n$ (or GWPA, for short) where $X=\left(X_{1}, \ldots, X_{n}\right)$ and $Y=\left(Y_{1}, \ldots, Y_{n}\right)$.

Existence of generalized Weyl Poisson algebras is proven in Sect. 2 (Lemma 2.1). The key idea of the proof is to introduce another class of Poisson algebras, elements of which are denoted by $D[X, Y ; \partial, \alpha]$ (see Sect. 2), for which existence problem has an easy solution and then to show that each GWPA is a factor algebra of some $D[X, Y ; \partial, \alpha]$. The Poisson algebras $D[X, Y ; \partial, \alpha]$ turn out to be also GWPAs (Proposition 2.2).

Poisson simplicity criterion for generalized Weyl Poisson algebras. A Poisson algebra is a simple Poisson algebra if the ideals 0 and $A$ of the associative algebra $A$ are the only ideals $I$ such that $\{A, I\} \subseteq I$. The ideal $I$ is called a Poisson ideal of the Poisson algebra $A$. An ideal $I$ of the ring $D$ is called $\partial$-invariant, where $\partial=\left(\partial_{1}, \ldots, \partial_{n}\right) \in \operatorname{PDer}_{K}(D)^{n}$, if $\partial_{i}(I) \subseteq I$ for all $i=1, \ldots, n$. The set $D^{\partial}:=\left\{d \in D \mid \partial_{1}(d)=0, \ldots, \partial_{n}(d)=0\right\}$ is called the ring of $\partial$-constants of D.

In Sect. 3, a proof is given of the following Poisson simplicity criterion for generalized Weyl Poisson algebras; see Proposition 3.1 for the notation.

Theorem 1.1 Let $A=D[X, Y ; a, \partial\}$ be a GWPA of rank $n$. Then, the Poisson algebra $A$ is a simple Poisson algebra iff

1. the Poisson algebra $D$ has no proper d-invariant Poisson ideals,

2. for all $i=1, \ldots, n, D a_{i}+D \partial_{i}\left(a_{i}\right)=D$, and

3. the algebra $\mathrm{PZ}(A)$ is a field, i.e. $\operatorname{char}(K)=0, \mathrm{PZ}(D)^{\partial}$ is a field and $D_{\alpha}=0$ for all $\alpha \in \mathbb{Z}^{n} \backslash\{0\}$ (see the proposition below).

As a first step in the proof of Theorem 1.1, the following field criterion for the Poisson centre $\mathrm{PZ}(A)$ of a GWPA $A=D[X, Y ; a, \partial\}$ of rank $n$ is proven (in Sect. 3).

Proposition 1.2 Let $A=D[X, Y ;$ a, $\partial\}$ be a GWPA of rankn. Then, $\mathrm{PZ}(A)$ is a field iff $\operatorname{char}(K)=0, \mathrm{PZ}(D)^{\partial}$ is a field and $D_{\alpha}=0$ for all $\alpha=\left(\alpha_{1}, \ldots, \alpha_{n}\right) \in \mathbb{Z}^{n} \backslash\{0\}$ where $D_{\alpha}=\left\{\lambda \in D^{\partial} \mid \operatorname{pad}_{\lambda}:=\{\lambda, \cdot\}=\lambda \sum_{i=1}^{n} \alpha_{i} \partial_{i}, \lambda \alpha_{i} \partial_{i}\left(a_{i}\right)=0\right.$ for $\left.i=1, \ldots, n\right\}$.

An explicit description of the Poisson centre is obtained (Proposition 3.1). Many examples are considered. We show that many classical Poisson algebras are GWPAs.

At the end of Sect. 2, we show that GWPAs appear as associated graded Poisson algebras of certain GWAs (Proposition 2.3). This is a sort of quantization procedure.

At the end of Sect. 3, examples of simple GWPAs (as Poisson algebras) are considered (Corollary 3.5). This family of simple Poisson algebras includes, as a particular case, the classical Poisson polynomial algebras $P_{2 n}=K\left[X_{1}, \ldots, X_{n}, Y_{1}, \ldots, Y_{n}\right]$ $\left(\left\{Y_{i}, X_{j}\right\}=\delta_{i j}\right.$ and $\left\{X_{i}, X_{j}\right\}=\left\{X_{i}, Y_{j}\right\}=\left\{Y_{i}, Y_{j}\right\}=0$ for all $\left.i \neq j\right)$. 


\section{The generalized Weyl Poisson algebras}

In this section, two new classes of Poisson algebras are introduced and their existence is proved. One of them is the class of generalized Weyl Poisson algebras (GWPAs). Examples are considered. At the end of the section, it is shown that some GWPAs are obtained from GWAs by a sort of quantization procedure (Proposition 2.3).

Poisson algebras. A commutative associative algebra $D$ is called a Poisson algebra if it is a Lie algebra $(D,\{\cdot, \cdot\})$ such that $\{a, x y\}=\{a, x\} y+x\{a, y\}$ for all elements $a, x, y \in D$.

For a $K$-algebra $D$, let $\operatorname{Der}_{K}(D)$ be the set of its $K$-derivations. If, in addition, $(D,\{\cdot, \cdot\})$ is a Poisson algebra, then

$\operatorname{PDer}_{K}(D):=\left\{\delta \in \operatorname{Der}_{K}(D) \mid \delta(\{a, b\})=\{\delta(a), b\}+\{a, \delta(b)\}\right.$ for all $\left.a, b \in D\right\}$

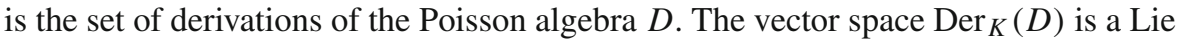
algebra, where $[\delta, \partial]:=\delta \partial-\partial \delta$, and $\operatorname{PDer}_{K}(D)$ is a Lie subalgebra of $\operatorname{Der}_{k}(D)$. The set of inner derivations

$$
\operatorname{IDer}_{K}(D):=\left\{\operatorname{ad}_{a} \mid a \in D\right\} \quad\left(\text { where } \operatorname{ad}_{a}(b):=[a, b]:=a b-b a\right)
$$

is an ideal of the Lie algebra $\operatorname{Der}_{K}(D)\left(\right.$ since $\left[\delta, \operatorname{ad}_{a}\right]=\operatorname{ad}_{\delta(a)}$ for all $\delta \in \operatorname{Der}_{K}(D)$ and $a \in D)$. Similarly, the set of inner derivations of the Poisson algebra $D$

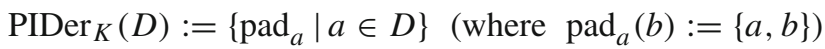

is an ideal of the Lie algebra $\operatorname{PDer}_{K}(D)\left(\right.$ since $\left[\delta, \operatorname{pad}_{a}\right]=\operatorname{pad}_{\delta(a)}$ for all $\delta \in$ $\operatorname{PDer}_{K}(D)$ and $\left.a \in D\right)$. By the very definition, the Poisson algebra $D$ is a Lie algebra with respect to the bracket $\{\cdot, \cdot\}$. The map $D \rightarrow \operatorname{PIDer}_{K}(D), a \mapsto \operatorname{pad}_{a}$, is an epimorphism of Lie algebras with kernel

$$
\mathrm{PZ}(D):=\{a \in D \mid\{a, D\}=0\}
$$

which is called the centre of the Poison algebra (or the Poisson centre of $D$ ). So, the Poisson structure of the algebra $D$ induces the 'multiplicative structure' on the Lie algebra $\operatorname{PIDer}_{K}(D)$, i.e. $\operatorname{pad}_{a b}(\cdot)=\operatorname{pad}_{a}(\cdot) b+a \operatorname{pad}_{b}(\cdot)$.

Notice that the centre $Z(D):=\{z \in D \mid z d=d z$ for all $d \in D\}$ of any associative algebra $D$ is invariant under the action of $\operatorname{Der}_{K}(D)$ : Let $z \in Z(D), d \in D$ and $\partial \in \operatorname{Der}_{K}(D)$; then, applying the derivation $\partial$ to the equality $z d=d z$, we obtain the equality $\partial(z) d=d \partial(z)$, i.e. $\partial(z) \in Z(D)$. Similarly, the Poisson centre PZ $(D)$ is invariant under the action of $\operatorname{PDer}_{K}(D)$ : Let $z \in \operatorname{PZ}(D), d \in D$ and $\partial \in \operatorname{PDer}_{K}(D)$; then, applying the derivation $\partial$ to the equality $\{z, d\}=0$, we obtain the equality $\{\partial(z), d\}=0$, i.e. $\partial(z) \in \mathrm{PZ}(D)$.

Let $D$ be a Poisson algebra, $\partial=\left(\partial_{1}, \ldots, \partial_{n}\right) \in \operatorname{PDer}_{K}(D)^{n}$ be an $n$-tuple of commuting derivations of the Poisson algebra $D$ and $X=\left(X_{1}, \ldots, X_{n}\right)$ be an $n$ tuple of commuting variables. The polynomial algebra $D[X]=D\left[X_{1}, \ldots, X_{n}\right]$ with coefficients from $D$ admits a Poisson structure which is an extension of the Poisson structure on $D$ given by the rule 


$$
\left\{X_{i}, X_{j}\right\}=0 \text { and }\left\{X_{i}, d\right\}=\partial_{i}(d) X_{i} \text { for } 1 \leq i, j \leq n \text { and } d \in D
$$

The Poisson algebra $D[X]$ is denoted by $D[X ; \partial]$ and is called the Poisson Ore extension of $D$ of rank $n$.

Let $G$ be a monoid. Suppose that the associative algebra $D=\oplus_{g \in G} D_{g}$ is a $G$ graded algebra $\left(D_{g} D_{h} \subseteq D_{g h}\right.$ for all $\left.g, h \in G\right)$. If, in addition, $D$ is a Poisson algebra and $\left\{D_{g}, D_{h}\right\} \subseteq D_{g h}$ for all $g, h \in G$, then we say that the Poisson algebra $D$ is a $G$-graded Poisson algebra.

The Poisson algebra $D[X, Y ; \partial, \alpha]$. Now, we introduce a class of Poisson algebras which is used in the proof of existence of GWPAs (Lemma 2.1).

Definition Let $D$ be a Poisson algebra, $\partial=\left(\partial_{1}, \ldots, \partial_{n}\right) \in \operatorname{PDer}_{K}(D)^{n}$ be an $n$ tuple of commuting derivations of the Poisson algebra $D$ and $\alpha=\left(\alpha_{1}, \ldots, \alpha_{n}\right) \in$ $\mathrm{PZ}(D)^{n}$. Then, the polynomial algebra $D[X, Y]=D\left[X_{1}, \ldots, X_{n}, Y_{1}, \ldots, Y_{n}\right]$ with coefficients in $D$ admits a Poisson structure which is an extension of the Poisson structure on $D$ given by the rule: For all $i, j=1, \ldots, n$ and $d \in D$,

$$
\begin{aligned}
\left\{Y_{i}, d\right\} & =\partial_{i}(d) Y_{i}, \quad\left\{X_{i}, d\right\}=-\partial_{i}(d) X_{i} \text { and }\left\{Y_{i}, X_{i}\right\}=\alpha_{i}, \\
\left\{X_{i}, X_{j}\right\} & =\left\{X_{i}, Y_{j}\right\}=\left\{Y_{i}, Y_{j}\right\}=0 \text { for all } i \neq j
\end{aligned}
$$

The Poisson algebra $D[X, Y]$ is denoted by $\mathcal{A}=D[X, Y ; \partial, \alpha]$ where $X=$ $\left(X_{1}, \ldots, X_{n}\right)$ and $Y=\left(Y_{1}, \ldots, Y_{n}\right)$.

Let us show that the Poisson structure on the polynomial algebra $D[X, Y]$ is well defined. Let $n=1$. The Poisson algebra $D\left[X_{1}, Y_{1} ; \partial_{1}, \alpha_{1}\right]$ is an extension of the Poisson Ore extension $D\left[X_{1} ;-\partial_{1}\right]$ by adding a commuting variable $Y_{1}$ where the Poisson structure on the algebra $D\left[X_{1}\right]\left[Y_{1}\right]$ is given by the rule

$$
\left\{Y_{1}, d\right\}=\partial_{1}(d) Y_{1} \text { and }\left\{Y_{1}, X_{1}\right\}=\alpha_{1}
$$

The Poisson structure on the algebra $D\left[X_{1}\right]\left[Y_{1}\right]$ is well defined as $\left\{Y_{1}, \cdot\right\}$ respects the relation $\left\{X_{1}, d\right\}=-\partial_{1}(d) X_{1}$ for all $d \in D$ :

$$
\begin{aligned}
\left\{Y_{1},\left\{X_{1}, d\right\}\right\} & =\left\{\alpha_{1}, d\right\}+\left\{X_{1}, \partial_{1}(d) Y_{1}\right\}=0-\partial_{1}^{2}(d) X_{1} Y_{1}-\partial_{1}(d) \alpha_{1} \\
& =-\left\{Y_{1}, \partial_{1}(d) X_{1}\right\} .
\end{aligned}
$$

For $n \geq 1$, the Poisson algebra

$$
D[X, Y ; \partial, \alpha]=D\left[X_{1}, Y_{1} ; \partial_{1}, \alpha_{1}\right] \cdots\left[X_{n}, Y_{n} ; \partial_{n}, \alpha_{n}\right]
$$

is an iteration of this construction $n$ times.

Consistency of the defining relations of generalized Weyl Poisson algebra follows from the next lemma.

Lemma 2.1 We keep the assumptions of the Definition of GWPA $A=D[X, Y ; a, \partial\}$. Let $\mathcal{A}=D[X, Y ; \partial, \partial(a)]$ where $\partial(a)=\left(\partial_{1}\left(a_{1}\right), \ldots, \partial_{n}\left(a_{n}\right)\right)$. Then, $X_{1} Y_{1}-$ 
$a_{1}, \ldots, X_{n} Y_{n}-a_{n} \in \operatorname{PZ}(\mathcal{A})$ and the generalized Weyl Poisson algebra $A=$ $D[X, Y ; a, \partial\}$ is a factor algebra of the Poisson algebra $\mathcal{A}$,

$$
A \simeq \mathcal{A} /\left(X_{1} Y_{1}-a_{1}, \ldots, X_{n} Y_{n}-a_{n}\right) .
$$

Proof By the very definition, the element $Z_{i}=X_{i} Y_{i}-a_{i} \in \mathrm{PZ}(A)$ : For all $i, j$ such that $i \neq j,\left\{X_{j}, Z_{i}\right\}=\partial_{j}\left(a_{i}\right) X_{j}=0$ and $\left\{Y_{j}, Z_{i}\right\}=-\partial_{j}\left(a_{i}\right) Y_{j}=0$ (since $\partial_{j}\left(a_{i}\right)=0$ for all $\left.i \neq j\right)$. For all $d \in D$,

$$
\begin{aligned}
\left\{Z_{i}, d\right\} & =\left\{X_{i}, d\right\} Y_{i}+X_{i}\left\{d, Y_{i}\right\}=-\partial_{i}(d) X_{i} Y_{i}+X_{i} \partial(d) Y_{i}=0, \\
\left\{X_{i}, Z_{i}\right\} & =X_{i}\left(-\partial_{i}\left(a_{i}\right)\right)+\partial_{i}\left(a_{i}\right) X_{i}=0, \\
\left\{Y_{i}, Z_{i}\right\} & =\partial_{i}\left(a_{i}\right) Y_{i}-\partial_{i}\left(a_{i}\right) Y_{i}=0 .
\end{aligned}
$$

Therefore, $Z_{i} \in \mathrm{PZ}(\mathcal{A})$. Now, the lemma is obvious.

$A \mathbb{Z}^{n}$-grading of a GWPA $A=D[X, Y ; a, \partial\}$. The GWPA of rank $n$,

$$
A:=D[X, Y ; a, \partial\}=\bigoplus_{\alpha \in \mathbb{Z}^{n}} A_{\alpha},
$$

is a $\mathbb{Z}^{n}$-graded Poisson algebra where $A_{\alpha}=D v_{\alpha}, v_{\alpha}=\prod_{i=1}^{n} v_{\alpha_{i}}(i)$ and

$$
v_{j}(i)= \begin{cases}X_{i}^{j} & \text { if } j>0 \\ 1 & \text { if } j=0 \\ Y_{i}^{|j|} & \text { if } j<0\end{cases}
$$

So, $A_{\alpha} A_{\beta} \subseteq A_{\alpha+\beta}$ and $\left\{A_{\alpha}, A_{\beta}\right\} \subseteq A_{\alpha+\beta}$ for all elements $\alpha, \beta \in \mathbb{Z}^{n}$.

The isomorphisms $s_{I}$ where $I \subseteq\{1, \ldots, n\}$ of GWPAs of rank $n$. Let $A=$ $D\left[X_{1}, Y_{1} ; a_{1}, \partial_{1}\right\}$ be a GWPA of rank 1. Clearly, $A \simeq D\left[Y_{1}, X_{1} ; a_{1},-\partial_{1}\right\}$, i.e. the $D$-homomorphism of Poisson algebras

$$
\begin{aligned}
& s_{1}: A=D\left[X_{1}, Y_{1} ; a_{1}, \partial_{1}\right\} \rightarrow D\left[Y_{1}, X_{1} ; a_{1},-\partial_{1}\right\} \\
& X_{1} \mapsto Y_{1}, \quad Y_{1} \mapsto X_{1}, \\
& d \mapsto d(d \in D)
\end{aligned}
$$

is an isomorphism. Similarly, let $A=D[X, Y ; a, \partial\}$ be a GWPA of rank $n \geq 1$ and $I$ be a subset of the set $\{1, \ldots, n\}$. Let $s_{I}$ be a bijection of the set $X \cup Y=$ $\left\{X_{1}, \ldots, X_{1}, Y_{1}, \ldots, Y_{n}\right\}$ which is given by the rule

$$
s_{I}\left(X_{i}\right)=\left\{\begin{array}{ll}
Y_{i} & \text { if } i \in I, \\
X_{i} & \text { if } i \notin I,
\end{array} \text { and } s_{I}\left(Y_{i}\right)= \begin{cases}X_{i} & \text { if } i \in I, \\
Y_{i} & \text { if } i \notin I .\end{cases}\right.
$$

Let $\operatorname{sign}(I) \partial:=\left(\varepsilon_{1} \partial_{1}, \ldots, \varepsilon_{n} \partial_{n}\right)$ where $\varepsilon_{i}=\left\{\begin{array}{ll}-1 & \text { if } i \in I, \\ 1 & \text { if } i \notin I .\end{array}\right.$ Then, the $D$ homomorphism of Poisson algebras 


$$
\begin{aligned}
& s_{I}: A \rightarrow D\left[s_{I}(X), s_{I}(Y) ; a, \operatorname{sign}(I) \partial\right\} \\
& X_{i} \mapsto s_{I}\left(X_{i}\right), \quad Y_{i} \mapsto s_{I}\left(Y_{i}\right), \\
& d \mapsto d(d \in D),
\end{aligned}
$$

is an isomorphism.

Recall that $\delta_{i j}$ is the Kronecker delta function. The next proposition shows that the Poisson algebras $D[X, Y ; \partial, \alpha]$ are GWPAs.

Proposition 2.2 The Poisson algebra $\mathcal{A}=D[X, Y ; \partial, \alpha]$ is a GWPA of rank $n$

$$
D\left[H_{1}, \ldots, H_{n}\right][X, Y ; H, \partial\}
$$

where $D\left[H_{1}, \ldots, H_{n}\right]$ is a Poisson polynomial algebra over $D$ such that $\left\{H_{i}, D\right\}=0$ and $\left\{H_{i}, H_{j}\right\}=0$ for all $i, j, H=\left(H_{1}, \ldots, H_{n}\right)$ and $\partial_{i}\left(H_{j}\right)=\delta_{i j} \alpha_{j} H_{j}$ for all $i, j$.

Proof Consider the following elements of the polynomial algebra $\mathcal{A}=D[X, Y]$,

$$
H_{1}=X_{1} Y_{1}, \ldots, H_{n}=X_{n} Y_{n} .
$$

Then, $\left\{H_{i}, D\right\}=0$ and $\left\{H_{i}, H_{j}\right\}=0$ for all $i, j$. So, the elements $H_{1}, \ldots, H_{n}$ belong to the Poisson centre of the Poisson algebra $\mathcal{D}=D\left[H_{1}, \ldots, H_{n}\right]$. Let $A=$ $D\left[H_{1}, \ldots, H_{n}\right][X, Y ; H, \partial\}$. It follows from the defining relations of the Poisson algebras $\mathcal{A}$ and $A$ that there is an epimorphism $\mathcal{A} \rightarrow A$ of Poisson algebras given by the rule $X_{i} \mapsto X_{i}, Y_{i} \mapsto Y_{i}, d \mapsto d$ where $d \in D$ (since $X_{i} Y_{i} \mapsto H_{i}$ ) which is clearly a bijection (it is the 'identity map' of associative algebras when we identify $X_{i} Y_{i}$ with $H_{i}$ ).

By Proposition 2.2, the Poisson algebra $\mathcal{A}=D[X, Y ; \partial, \alpha]=\oplus_{\beta \in \mathbb{Z}^{n}} \mathcal{A}_{\beta}$ is $\mathbb{Z}^{n}$ $\operatorname{graded}\left(\mathcal{A}_{\beta} \mathcal{A}_{\gamma} \subseteq \mathcal{A}_{\beta+\gamma}\right.$ and $\left\{\mathcal{A}_{\beta}, \mathcal{A}_{\gamma}\right\} \subseteq \mathcal{A}_{\beta+\gamma}$ for all $\left.\beta, \gamma \in \mathbb{Z}^{n}\right)$ where $\mathcal{A}_{\beta}=\mathcal{D} v_{\beta}$, $\mathcal{D}=D\left[H_{1}, \ldots, H_{n}\right]$ and $v_{\beta}=\prod_{i=1}^{n} v_{\beta_{i}}(i)$ where $v_{j}(i)= \begin{cases}X_{i}^{j} & \text { if } j>0, \\ 1 & \text { if } j=0, \\ Y_{i}^{|j|} & \text { if } j<0 .\end{cases}$

Examples of GWPAs 1. If $D$ is a algebra with trivial Poisson bracket, then any choice of elements $a=\left(a_{1}, \ldots, a_{n}\right)$ and $\partial=\left(\partial_{1}, \ldots, \partial_{n}\right) \in \operatorname{Der}_{K}(D)^{n}$ such that $\partial_{i}\left(a_{j}\right)=0$ for all $i \neq j$ determines a GWPA $D[X, Y ; a, \partial\}$ of rank $n$. If, in addition, $n=1$, then there is no restriction on $a_{1}$ and $\partial_{1}$.

2. The classical Poisson polynomial algebra $P_{2 n}=K\left[X_{1}, \ldots, X_{n}, Y_{1}, \ldots, Y_{n}\right]$ $\left(\left\{Y_{i}, X_{j}\right\}=\delta_{i j}\right.$ and $\left\{X_{i}, X_{j}\right\}=\left\{X_{i}, Y_{j}\right\}=\left\{Y_{i}, Y_{j}\right\}=0$ for all $\left.i \neq j\right)$ is a GWPA

$$
P_{2 n}=K\left[H_{1}, \ldots, H_{n}\right][X, Y ; a, \partial\}
$$

where $K\left[H_{1}, \ldots, H_{n}\right]$ is a Poisson polynomial algebra with trivial Poisson bracket, $a=\left(H_{1}, \ldots, H_{n}\right), \partial=\left(\partial_{1}, \ldots, \partial_{n}\right)$ and $\partial_{i}=\frac{\partial}{\partial_{H_{i}}}$ (via the isomorphism of Poisson algebras $\left.P_{2 n} \rightarrow K\left[H_{1}, \ldots, H_{n}\right][X, Y ; a, \partial\}, X_{i} \mapsto X_{i}, Y_{i} \mapsto Y_{i}\right)$.

3. $A=D[X, Y ; a, \partial\}$ where $D=K\left[H_{1}, \ldots, H_{n}\right]$ is a Poisson polynomial algebra with trivial Poisson bracket, $a=\left(a_{1}, \ldots, a_{n}\right) \in K\left[H_{1}\right] \times \cdots \times K\left[H_{n}\right]$, 
$\partial=\left(\partial_{1}, \ldots, \partial_{n}\right)$ where $\partial_{i}=b_{i} \partial_{H_{i}}\left(\right.$ where $\left.\partial_{H_{i}}=\frac{\partial}{\partial H_{i}}\right)$ and $b_{i} \in K\left[H_{i}\right]$. In particular, $D\left[X, Y ;\left(H_{1}, \ldots, H_{n}\right),\left(\partial_{H_{1}}, \ldots, \partial_{H_{n}}\right)\right\}=P_{2 n}$ is the classical Poisson polynomial algebra.

Let $S$ be a multiplicative set of $D$. Then, $S^{-1} A \simeq\left(S^{-1} D\right)[X, Y ; a, \partial\}$ is a GWPA. In particular, for $S=\left\{H^{\alpha} \mid \alpha \in \mathbb{Z}^{n}\right\}$, we have $K\left[H_{1}^{ \pm 1}, \ldots, H_{n}^{ \pm 1}\right][X, Y ; a, \partial\}$. In the case $n=1$, the Poisson algebra

$$
K\left[H_{1}^{ \pm 1}\right]\left[X_{1}, Y_{1} ; a_{1},-H_{1} \frac{d}{d H_{1}}\right\}
$$

where $a_{1} \in K\left[H_{1}^{ \pm 1}\right]$ is, in fact, isomorphic to a Poisson algebra in the paper of Cho and $\mathrm{Oh}$ [4] which is obtained as a quantization of a certain GWA with respect to the quantum parameter $q$. In [4, Theorem 3.7], a Poisson simplicity criterion is given for this Poisson algebra.

4. Let $D=K[C, H]$ be a Poisson polynomial algebra with trivial Poisson bracket, $a \in D$ and $\partial$ is a derivation of the algebra $D$. The GWPA $A=D[X, Y ; a, \partial\}$ of rank 1 is a generalization of some Poisson algebras that are associated with $U\left(\operatorname{sl}_{2}\right)$, see the next example.

5. Let $U=U\left(\mathrm{sl}_{2}\right)$ be the universal enveloping algebra of the Lie algebra

$$
\mathrm{sl}_{2}=K\langle X, Y, H \mid[H, X]=X, \quad[H, Y]=-Y, \quad[X, Y]=2 H\rangle
$$

over a field $K$ of characteristic zero. The associated graded algebra $\operatorname{gr}(U)$ with respect to the filtration $\mathcal{F}=\left\{\mathcal{F}_{i}\right\}_{i \in \mathbb{N}}$ that is determined by the total degree of the elements $X$, $Y$ and $H$ is a Poisson polynomial algebra $K[X, Y, H]$ where

$$
\{H, X\}=X, \quad\{X, Y\}=-Y \text { and }\{X, Y\}=2 H .
$$

The element $C=X Y+H^{2}$ belongs to the Poisson centre of the Poisson polynomial algebra $\operatorname{gr}(U)$. The Poisson algebra

$$
\operatorname{gr}(U)=K[C, H]\left[X, Y ; a=C-H^{2}, \partial_{H}\right\}
$$

is a GWPA of rank 1 where $\partial_{H}:=\frac{\partial}{\partial H}$.

6. Let $U$ be the universal enveloping algebra of the Heisenberg Lie algebra

$$
\begin{aligned}
\mathcal{H}_{n}= & K\left\langle X_{1}, \ldots, X_{n}, Y_{1}, \ldots, Y_{n}, Z\right|\left[X_{i}, Y_{j}\right]=\delta_{i j} Z,\left[X_{i}, X_{j}\right]=\left[Y_{i}, Y_{j}\right]=0 \text { for all } i, j ; \\
& Z \text { is a Poisson central element }\rangle .
\end{aligned}
$$

The associated graded algebra $\operatorname{gr}(U)$ with respect to the filtration by the total degree of the canonical generators is a Poisson polynomial algebra $K\left[X_{1}, \ldots, X_{n}, Y_{1}, \ldots\right.$, $\left.Y_{n}, Z\right]$ where, for all $i, j$,

$$
\left\{X_{i}, Y_{j}\right\}=\delta_{i j} Z, \quad\left\{X_{i}, X_{j}\right\}=\left\{Y_{i}, Y_{j}\right\}=0
$$

and the element $Z$ belongs to the Poisson centre of $\operatorname{gr}(U)$. Then, the polynomial algebra 


$$
\operatorname{gr}(U)=D[X, Y ; a, \partial\}
$$

is a GWPA of rank $n$ where $D=K\left[H_{1}, \ldots, H_{n}, Z\right]$ is a Poisson polynomial algebra with trivial Poisson bracket, $X=\left(X_{1}, \ldots, X_{n}\right), Y=\left(Y_{1}, \ldots, Y_{n}\right), a=\left(a_{1}=\right.$ $\left.H_{1}, \ldots, a_{n}=H_{n}\right), \partial=\left(Z \partial_{H_{1}}, \ldots, Z \partial_{H_{n}}\right)$ and $\partial_{H_{i}}:=\frac{\partial}{\partial H_{i}}$.

Let $A_{s}=D_{s}\left[X_{(s)}, Y_{(s)} ; a_{(s)}, \partial_{(s)}\right\}$ be GWPAs of rank $n_{s}$ where $s=1, \ldots, m$. The tensor product of algebras

$$
A=\bigotimes_{s=1}^{m} A_{s}=\left(\bigotimes_{s=1}^{m} D_{s}\right)[X, Y ; a, \partial\}
$$

is a GWPA of rank $n_{1}+\cdots+n_{m}$ where $X=\left(X_{(1)}, \ldots, X_{(m)}\right), Y=\left(Y_{(1)}, \ldots, Y_{(m)}\right)$, $a=\left(a_{(1)}, \ldots, a_{(m)}\right)$ and $\partial=\left(\partial_{(1)}, \ldots, \partial_{(m)}\right)$. The Poisson structure on $A$ is a tensor product of Poisson structures on $A_{s}$, i.e. for all elements $u=\otimes_{s=1}^{m} u_{s}, v=\otimes_{s=1}^{m} v_{s} \in A$ (where $u_{s}, v_{s} \in A_{s}$ ),

$$
\{u, v\}=\sum_{s=1}^{m} u_{1} v_{1} \otimes \cdots \otimes\left\{u_{s}, v_{s}\right\} \otimes \cdots \otimes u_{m} v_{m} .
$$

Example The classical Poisson polynomial algebra $P_{2 n}$ [see (10)] is the tensor product $P_{2}^{\otimes n}$ of $n$ copies of the classical Poisson polynomial algebra $P_{2}$.

An algebraic torus action on a GWPA Let $A=D[X, Y ; a, \partial\}$ be a GWPA of rank $n$ and $\operatorname{AutPois}_{(A)}($ be the group of automorphisms of the Poisson algebra $A$. Elements of $\operatorname{Aut}_{\mathrm{Pois}}(A)$ are called Poisson automorphisms of $A$. For each element $\lambda=\left(\lambda_{1}, \ldots, \lambda_{n}\right) \in K^{* n}$, the $K$-algebra homomorphism

$$
t_{\lambda}: A \rightarrow A, \quad X_{i} \mapsto \lambda_{i} X_{i}, \quad Y_{i} \mapsto \lambda_{i}^{-1} Y_{i}, \quad d \mapsto d(d \in D),
$$

is an automorphism of the Poisson algebra $A$. The subgroup $\mathbb{T}^{n}=\left\{t_{\lambda} \mid \lambda \in K^{* n}\right\}$ of $\operatorname{Aut}_{\text {Pois }}(A)$ is an algebraic torus $\mathbb{T}^{n} \simeq K^{* n}, t_{\lambda} \mapsto \lambda$. For all $\alpha \in \mathbb{Z}^{n}$ and $u_{\alpha} \in A_{\alpha}=$ $D v_{\alpha}, t_{\lambda}\left(u_{\alpha}\right)=\lambda^{\alpha} \cdot u_{\alpha}$ where $\lambda^{\alpha}=\prod_{i=1}^{n} \lambda_{i}^{\alpha_{i}}$.

The subgroup

$\operatorname{Aut}_{\text {Pois }}(D)^{\partial, a}:=\left\{\sigma \in \operatorname{Aut}_{\text {Pois }}(D) \mid \sigma \partial_{i}=\partial_{i} \sigma\right.$ and $\sigma\left(a_{i}\right)=a_{i}$ for $\left.i=1, \ldots, n\right\}$

of $\operatorname{Aut}_{\text {Pois }}(D)$ can be seen as a subgroup of $\operatorname{Aut}_{\text {Pois }}(A)$ where each automorphism $\sigma \in \operatorname{Aut}_{\text {Pois }}^{\partial, a}(D)$ trivially acts at $X$ and $Y$, i.e. $\sigma\left(X_{i}\right)=X_{i}$ and $\sigma\left(Y_{i}\right)=Y_{i}$. Clearly,

$$
\mathbb{T}^{n} \times \operatorname{Aut}_{\text {Pois }}(D)^{\partial, a} \subseteq \operatorname{Aut}_{\text {Pois }}(A) .
$$

Associated graded algebra of a GWA is a GWPA. Let $A=D[X, Y ; \sigma, a]$ be a GWA of rank $n$ such that $D=\cup_{i \in \mathbb{N}} D_{i}$ is a filtered algebra $\left(D_{i} D_{j} \subseteq D_{i+j}\right.$ for all $i, j \in \mathbb{N}$; $\left.D_{-1}=0\right)$,

$\left[d_{i}, d_{j}\right] \in D_{i+j-\nu}$ for all $d_{i} \in D_{i}$ and $d_{j} \in D_{j}$ where $v$ is a positive integer; 
$\sigma_{i}\left(D_{j}\right)=D_{j}$ and $\left(\sigma_{i}-1\right)\left(D_{j}\right) \subseteq D_{j-v}$ for all $i=1, \ldots, n$ and $j \in \mathbb{N}$. Suppose that $a_{i} \in D_{d_{i}} \backslash D_{d_{i}-1}$ for some $d_{i} \geq 1$. The algebra $A$ admits a filtration $\left\{A_{s}\right\}_{s \in \frac{1}{2} \mathbb{N}}$ where

$$
\begin{aligned}
A_{s} & =\sum_{i+d \cdot \alpha \leq s} D_{i} v_{\alpha}, \quad d=\left(d_{1}, \ldots, d_{n}\right), \quad \alpha=\left(\alpha_{1}, \ldots, \alpha_{n}\right) \in \mathbb{Z}^{n} \text { and } d \cdot \alpha \\
& =\frac{1}{2} \sum_{i=1}^{n} d_{i}\left|\alpha_{i}\right| .
\end{aligned}
$$

The associated graded algebra

$$
\begin{aligned}
\operatorname{gr}(A) & =\operatorname{gr}(D)[X, Y ;(\mathrm{id}, \ldots, \mathrm{id}), \bar{a}] \\
& =\operatorname{gr}(D)\left[X_{1}, \ldots, X_{n}, Y_{1}, \ldots, Y_{n}\right] /\left(X_{1} Y_{1}-\bar{a}_{1}, \ldots, X_{n} Y_{n}-\bar{a}_{n}\right)
\end{aligned}
$$

is a commutative GWA where $\bar{a}_{i}=a_{i}+D_{d_{i}-1} \in D_{d_{i}} / D_{d_{i}-1}$. For all elements $u_{s} \in A_{s}$ and $u_{t} \in A_{t}$,

$$
\left[u_{s}, u_{t}\right] \in A_{s+t-v} .
$$

Let $\bar{u}_{s}=u_{s}+A_{s-1} \in A_{s} / A_{s-1}$ and $\bar{u}_{t}=u_{t}+A_{t-1} \in A_{t} / A_{t-1}$. The bracket

$$
\left\{u_{s}, u_{t}\right\}:=\overline{\left[u_{s}, u_{t}\right]}:=\left[u_{s}, u_{t}\right]+A_{s+t-v-1} \in A_{s+t-v} / A_{s+t-v-1}
$$

determines the Poisson structure on $\operatorname{gr}(A)$. For each $i=1, \ldots, n$, the map

$$
\partial_{i}:=\overline{\sigma_{i}-1}: \operatorname{gr}(D) \rightarrow \operatorname{gr}(D), \quad \operatorname{gr}(D)_{j} \ni \bar{b}_{j} \mapsto\left(\sigma_{i}-1\right)\left(b_{j}\right)+D_{j-v-1} \in \operatorname{gr}(D)_{j-v},
$$

is a $K$-derivation of the commutative algebra $\operatorname{gr}(D)$. The derivations $\partial_{1}, \ldots, \partial_{n}$ commute since the automorphisms $\sigma_{1}, \ldots, \sigma_{n}$ commute. Notice that

$$
\left[X_{i}, b_{j}\right]=\left(\sigma_{i}-1\right)\left(b_{j}\right) X_{i} \text { and }\left[Y_{i}, b_{j}\right]=\left(\sigma_{i}^{-1}-1\right)\left(b_{j}\right) Y_{i}
$$

Hence, $\left\{X_{i}, \bar{b}_{j}\right\}=\partial_{i}\left(b_{j}\right) X_{i}$ and $\left\{Y_{i}, \bar{b}_{j}\right\}=-\partial_{i}\left(b_{j}\right) Y_{i}$ since

$$
\left(\sigma_{i}^{-1}-1\right)\left(b_{j}\right)=-\left(\sigma_{i}-1\right) \sigma_{i}^{-1}\left(b_{j}\right) \equiv-\partial_{i}\left(\bar{b}_{j}\right) \quad \bmod D_{j-v-1}
$$

Therefore, the Poisson algebra $\operatorname{gr}(A)$ is a GWPA $\operatorname{gr}(D)[X, Y ; \bar{a},-\partial\}$ where $\bar{a}=$ $\left(\bar{a}_{1}, \ldots, \bar{a}_{n}\right)$ and $-\partial=\left(-\partial_{1}, \ldots,-\partial_{n}\right)$. So, we proved that the following proposition holds.

Proposition 2.3 Let $A=D[X, Y ; \sigma, a]$ be a GWA of rank $n$ such that $D=\cup_{i \in \mathbb{N}} D_{i}$ is a filtered algebra; $\left[d_{i}, d_{j}\right] \in D_{i+j-v}$ for all $d_{i} \in D_{i}$ and $d_{j} \in D_{j}$ where $v$ is a positive integer; $\sigma_{i}\left(D_{j}\right)=D_{j}$ and $\left(\sigma_{i}-1\right)\left(D_{j}\right) \subseteq D_{j-v}$ for all $i=1, \ldots, n$ and $j \in \mathbb{N}$. Suppose that $a_{i} \in D_{d_{i}} \backslash D_{d_{i}-1}$ for some $d_{i} \geq 1$. Let $\left\{A_{s}\right\}_{s \in \frac{1}{2} \mathbb{N}}$ be the filtration as above. The associated graded algebra $\operatorname{gr}(A)$ is a $G W P A \operatorname{gr}(D)[X, Y ; \bar{a},-\partial\}$ where $\bar{a}$ and $-\partial$ are defined above. 
Example 1 . The $n$ 'th Weyl algebra $A_{n}$ is a GWA $K\left[H_{1}, \ldots, H_{n}\right][X, Y ; \sigma, a]$ where $\sigma_{i}\left(H_{j}\right)=H_{j}-\delta_{i j}$ and $a_{i}=H_{i}$ for $i, j=1, \ldots, n$. The polynomial algebra $D=$ $K\left[H_{1}, \ldots, H_{n}\right]$ admits a natural filtration $\left\{D_{i}\right\}_{i \in \mathbb{N}}$ by the total degree of the variables $H_{1}, \ldots, H_{n}$. The automorphisms $\sigma_{1}, \ldots, \sigma_{n}$ satisfy the conditions of Proposition 2.3 with $v=1, d_{1}=\cdots=d_{n}=1$ and $\partial_{1}=-\frac{\partial}{\partial H_{1}}, \ldots, \partial_{n}=-\frac{\partial}{\partial H_{n}}$. Notice that $\operatorname{gr}(D)=D$. By Proposition 2.3, the algebra

$$
\operatorname{gr}\left(A_{n}\right) \simeq D[X, Y] /\left(X_{1} Y_{1}-H_{1}, \ldots, X_{n} Y_{n}-H_{n}\right) \simeq K[X, Y]=P_{2 n}
$$

is a GWPA $D\left[X, Y ;\left(H_{1}, \ldots, H_{n}\right),\left(\frac{\partial}{\partial H_{1}}, \ldots, \frac{\partial}{\partial H_{n}}\right)\right\}$ which is the classical Poisson algebra $P_{2 n}$ with the canonical Poisson bracket $\left(\left\{Y_{i}, X_{j}\right\}=\delta_{i j},\left\{X_{i}, X_{j}\right\}=\right.$ $\left\{X_{i}, Y_{j}\right\}=\left\{Y_{i}, Y_{j}\right\}=0$ for all $i, j$ such that $\left.i \neq j\right)$.

2. The universal enveloping algebra $U=U\left(\mathrm{sl}_{2}\right)$ is the GWA $A=K[C, H][X, Y$; $\sigma, a$ ] of rank 1 where $\sigma(H)=H-1, \sigma(C)=C$ and $a=C-H(H+1)$ (the element $C$ is the Casimir element, $C=Y X+H(H+1))$. The filtration $\mathcal{F}=\left\{\mathcal{F}_{i}\right\}_{i \in \mathbb{N}}$ on $U$ that was considered above (which is defined by the total degree of the canonical generators $X, Y$ and $H$ ) induces a filtration $\left\{D_{i}:=D \cap \mathcal{F}_{i}\right\}_{i \in \mathbb{N}}$ on the polynomial algebra $D=K[C, H]$. Clearly,

$$
D_{i}=\bigoplus_{2 s+t \leq i} K C^{s} H^{t} \text { for all } i \in \mathbb{N}
$$

The automorphism $\sigma$ and the filtration $\left\{D_{i}\right\}_{i \in \mathbb{N}}$ satisfy the conditions of Proposition 2.3 where $d_{1}=2$ and $v=-1$. The associated graded Poisson algebra $\operatorname{gr}(A) \simeq K[C, H]\left[X, Y ; C-H^{2}, \partial_{H}\right\}$ is canonically isomorphic to the associated graded Poisson algebra $\operatorname{gr}(U)$ as $\mathbb{N}$-graded Poisson algebra (since $\operatorname{gr}(A)_{\frac{1}{2}+i}=0$ for all $i \in \mathbb{N}$ ), see (11).

The filtration $\left\{D_{i}^{\prime}:=\oplus_{j \leq i} K[C] H^{i}\right\}_{i \in \mathbb{N}}$ also satisfies the conditions of Proposition 2.3 where $d_{1}=2$ and $v=-1$ but the associated graded algebra $\operatorname{gr}^{\prime}(A)$ is a GWPA $K[C, H]\left[X, Y ;-H^{2}, \partial_{H}\right\}$. The associated graded Poisson algebras $\operatorname{gr}(A)$ and $\operatorname{gr}^{\prime}(A)$ are not isomorphic since the algebra $\operatorname{gr}(A)$ is smooth but the algebra $\operatorname{gr}^{\prime}(A) \simeq K[C] \otimes K[X, Y]\left(X Y-H^{2}\right)$ is singular as the points $\{(C, H, X, Y)=$ $(\lambda, 0,0,0) \mid \lambda \in K\}$ are singular. So, the Poisson algebras $\operatorname{gr}(A)$ and $\operatorname{gr}^{\prime}(A)$ are also not isomorphic.

\section{Poisson simplicity criterion for generalized Weyl Poisson algebras}

In this section, for generalized Weyl Poisson algebras, a proof of the Poisson simplicity criterion (Theorem 1.1) is given, an explicit description of their Poisson centre is obtained (Proposition 3.1) and a proof of the criterion for the Poisson centre being a field (Proposition 1.2) is given.

Let $A$ be a Poisson algebra. An ideal $I$ of the associative algebra $A$ is called a Poisson ideal if $\{A, I\} \subseteq I$. A Poisson ideal is also called an ideal of the Poisson algebra. Suppose that $\mathcal{D}$ be a set of derivations of the associative algebra $A$. Then, the 
set $A^{\mathcal{D}}:=\{a \in A \mid \partial(a)=0$ for all $\partial \in \mathcal{D}\}$ is a subalgebra of $A$ which is called the algebra of $\mathcal{D}$-constants (or the algebra of constants for $\mathcal{D}$ ). An ideal $J$ of the algebra $A$ is called a $\mathcal{D}$-invariant ideal if $\partial(J) \subseteq J$ for all $\partial \in \mathcal{D}$.

The Poisson centre of a GWPA. Let $A=D[X, Y ; a, \partial\}$ be a GWPA of rank $n$. For all elements $\lambda, d \in D, \alpha \in \mathbb{Z}^{n}$ and $i=1, \ldots, n$

$$
\begin{aligned}
\left\{d, \lambda v_{\alpha}\right\} & =\left(-\operatorname{pad}_{\lambda}+\lambda \sum_{i=1}^{n} \alpha_{i} \partial_{i}\right)(d) v_{\alpha}, \\
\left\{v_{ \pm 1}(i), \lambda v_{\alpha}\right\} & = \begin{cases}\mp \partial_{i}(\lambda) v_{\alpha \pm e_{i}} & \text { if } \alpha_{i}=0 \text { or } \operatorname{sign}\left(\alpha_{i}\right)= \pm, \\
\left(\mp \partial_{i}(\lambda) a_{i}+\lambda \alpha_{i} \partial_{i}\left(a_{i}\right)\right) v_{\alpha \pm e_{i}} & \text { if } \operatorname{sign}\left(\alpha_{i}\right)=\mp .\end{cases}
\end{aligned}
$$

The next proposition describes the Poisson centre of a GWPA.

Proposition 3.1 Let $A=D[X, Y ; a$, $\partial\}$ be a GWPA of rank $n$. Then, $\mathrm{PZ}(A)=$ $\bigoplus_{\alpha \in \mathbb{Z}^{n}} \mathrm{PZ}(A)_{\alpha}$ is a $\mathbb{Z}^{n}$-graded (associative) algebra where $\mathrm{PZ}(A)_{\alpha}=D_{\alpha} v_{\alpha}, D_{0}=$ $\mathrm{PZ}(D)^{\partial}$ and, for all $\alpha \neq 0, D_{\alpha}=\left\{\lambda \in D^{\partial} \mid \operatorname{pad}_{\lambda}=\lambda \sum_{i=1}^{n} \alpha_{i} \partial_{i}, \lambda \alpha_{i} \partial_{i}\left(a_{i}\right)=0\right.$ for $i=1, \ldots, n\}$.

Proof The GWPA $A=\oplus_{\alpha \in \mathbb{Z}^{n}} A_{\alpha}$ is a $\mathbb{Z}^{n}$-graded Poisson algebra, hence so is its Poisson centre, i.e. $\mathrm{PZ}(A)=\bigoplus_{\alpha \in \mathbb{Z}^{n}} \mathrm{PZ}(A)_{\alpha}$ where $\mathrm{PZ}(A)_{\alpha}=\mathrm{PZ}(A) \cap A_{\alpha}$. Since $A_{\alpha}=D v_{\alpha}$ for all $\alpha \in \mathbb{Z}^{n}$, statement 2 follows from (16) and (17).

The next corollary shows that, in general, the Poisson centre of a GWPA $A$ is small.

Corollary 3.2 Let $A=D[X, Y ; a$, d $\}$ be a GWPA of rankn. Suppose that char $(K)=0$ and the elements $\partial_{1}\left(a_{1}\right), \ldots, \partial_{n}\left(a_{n}\right)$ are nonzero divisors in the algebra $D($ e.g. $D$ is a domain and $\left.\partial_{1}\left(a_{1}\right) \neq 0, \ldots, \partial_{n}\left(a_{n}\right) \neq 0\right)$. Then, $\mathrm{PZ}(A)=\mathrm{PZ}(D)^{\partial}$.

For an element $\alpha=\left(\alpha_{1}, \ldots, \alpha_{n}\right) \in \mathbb{Z}^{n}$, the set $\operatorname{supp}(\alpha):=\left\{i \mid \alpha_{i} \neq 0\right\}$ is called the support of $\alpha$.

Corollary 3.3 Let $A=D[X, Y ; a$, d $\}$ be a GWPA of rank $n$. Suppose that char $(K)=$ 0 . Then, for all elements $\alpha \in \mathbb{Z}^{n} \backslash\{0\}, D_{\alpha} \subseteq D^{\partial, \operatorname{pad}(\partial(a))} \cap \operatorname{ann}_{D}\left\{\partial_{i}\left(a_{i}\right) \mid i \in \operatorname{supp}(\alpha)\right\}$, i.e.

1. $\left\{D_{\alpha}, a_{i}\right\}=0$ for $i=1, \ldots, n$, and

2. $D_{\alpha} \partial_{i}\left(a_{i}\right)=0$ for all $i$ such that $\alpha_{i} \neq 0$.

Proof By Proposition 3.1.(3), $D_{\alpha} \partial_{i}\left(a_{i}\right)=0$ for all $i \in \operatorname{supp}(\alpha)(\operatorname{since} \operatorname{char}(K)=0)$. Then, for all $\lambda \in D_{\alpha}$ and $i=1, \ldots, n,\left\{\lambda, a_{i}\right\}=\operatorname{pad}_{\lambda}\left(a_{i}\right)=\sum_{i=1}^{n} \lambda \alpha_{i} \partial_{i}\left(a_{i}\right)=0$, i.e. $\left\{D_{\alpha}, a_{i}\right\}=0$ for $i=1, \ldots, n$.

Let $A=\bigoplus_{i \in \mathbb{Z}} A_{i}$ be a $\mathbb{Z}$-graded (associative) algebra. Each element $a \in A$ is a unique sum $a=\sum_{i \in \mathbb{Z}} a_{i}$ where $a_{i} \in A_{i}$. The length $l(a)$ of the element $a$ is equal to $-\infty$ if $a=0$, and, for $a \neq 0, l(a):=n-m$ where $n=\max \left\{i \mid a_{i} \neq 0\right\}$ and $m=\min \left\{i \mid a_{i} \neq 0\right\}$.

Let $A$ be a Poisson algebra and $z \in \operatorname{PZ}(A)$. The $z A$ is a Poisson ideal of $A$. If the Poisson algebra $A$ is simple, then necessarily the Poisson centre $\operatorname{PZ}(A)$ is a field. 
Proof of Proposition $1.2(\Rightarrow)$ Suppose that $p=\operatorname{char}(K) \neq 0$. Then, by Proposition 3.1, the element $1+X^{p}$ of $\mathrm{PZ}(A)$ is not invertible. Therefore, we must have $p=0$. The algebras $A$ and $\mathrm{PZ}(A)$ are $\mathbb{Z}^{\alpha}$-graded algebras and $\mathrm{PZ}(A)_{0}=\mathrm{PZ}(D)^{\partial}$. Therefore, $\mathrm{PZ}(D)^{\partial}$ must be a field.

Suppose that $D_{\alpha} \neq 0$ for some $\alpha \neq 0$. Then, $\alpha_{i} \neq 0$ for some $i$. Fix a nonzero element of $\mathrm{PZ}(A)_{\alpha}=D_{\alpha} v_{\alpha}$, say $\lambda v_{\alpha}$ where $\lambda \in D_{\alpha}$. Since $\lambda v_{\alpha}$ is a unit, $\left(\lambda v_{\alpha}\right)^{-1}=$ $\mu v_{-\alpha}$ (since the algebra $A$ is a $\mathbb{Z}^{n}$-graded algebra), and so

$$
1=\lambda v_{\alpha} \cdot \mu v_{-\alpha}=\lambda \mu a^{|\alpha|} \text { and } 1=\mu v_{-\alpha} \cdot \lambda v_{\alpha}=\mu \lambda a^{|\alpha|}
$$

where $a^{|\alpha|}:=\prod_{i=1}^{n} a_{i}^{\left|\alpha_{i}\right|} \in \mathrm{PZ}(A)$. Hence, $a^{|\alpha|}$ is a unit in $\mathrm{PZ}(A)$; then, the elements $\lambda$ and $\mu$ are units in $D$. Clearly, $v:=1+\lambda v_{\alpha} \in \mathrm{PZ}(A)$. The algebra $A$ is a $\mathbb{Z}^{n}$-graded algebra. In particular, it is a $\mathbb{Z} e_{i}$-graded algebra (since $\mathbb{Z} e_{i} \subseteq \mathbb{Z}^{n}$ ). Let $l_{i}$ be the length with respect to the $\mathbb{Z} e_{i}$-grading (which is a $\mathbb{Z}$-grading). Then, for all nonzero elements $u \in A$,

$$
l_{i}(u v)=l_{i}(u)+l_{i}(v) \geq l_{i}(v)=\left|\alpha_{i}\right|>0,
$$

since the elements 1 and $\lambda$ are units. This implies that the element $u$ is not a unit. Therefore, $D_{\alpha}=0$ for all $\alpha \in \mathbb{Z}^{n} \backslash\{0\}$, by Proposition 3.1.(3).

$(\Leftarrow)$ By Proposition 3.1, $\mathrm{PZ}(A)=\mathrm{PZ}(D)^{\partial}$ is a field.

An ideal $I$ of an algebra $A$ is called a proper ideal if $I \neq 0, A$.

Proof of Theorem $1.1(\Rightarrow)$ Suppose that $\mathfrak{a}$ is a proper $\partial$-invariant Poisson ideal of the

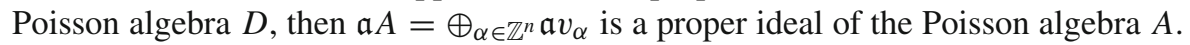
So, the first condition holds.

Suppose that $\mathfrak{b}:=D a_{i}+D \partial_{i}\left(a_{i}\right) \neq D$ for some $i$. Then,

$$
I=\bigoplus_{\alpha \in \mathbb{Z}^{n}, \alpha_{i} \neq 0} D v_{\alpha} \oplus \bigoplus_{\alpha \in \mathbb{Z}^{n}, \alpha_{i}=0} \mathfrak{b} v_{\alpha}
$$

is a proper ideal of the Poisson algebra $A$. So, the second condition holds.

The third condition obviously holds. (If a nonzero element $z$ of $\mathrm{PZ}(A)$ is also a nonunit, then $z A$ is a proper Poisson ideal of $A$ ).

$(\Leftarrow)$ Suppose that conditions 1 and 2 hold. Then, the implication follows from the Claim.

Claim. Suppose that conditions 1 and 2 hold. Then, every nonzero Poisson ideal of $A$ intersects nontrivially $\mathrm{PZ}(A)$.

Let $I$ be a nonzero Poisson ideal $A$. We have to show that $I \cap \operatorname{PZ}(A) \neq 0$. Let $u=\sum_{\alpha \in \mathbb{Z}^{n}} u_{\alpha}$ be a nonzero element of $I$ where $u_{\alpha} \in A_{\alpha}$. The set $\operatorname{supp}(u)=\{\alpha \in$ $\left.\mathbb{Z}^{n} \mid u_{\alpha} \neq 0\right\}$ is called the support of $u$. Recall that, for $\alpha \in \mathbb{Z}^{n},|\alpha|=\alpha_{1}+\cdots+\alpha_{n}$. The additive group $\mathbb{Z}^{n}$ admits the degree-by-lexicographic ordering $\leq$ where $\alpha<\beta$ iff either $|\alpha|<|\beta|$ or $|\alpha|=|\beta|$ and there exists an element $i \in\{1, \ldots, n\}$ such that $\alpha_{j}=\beta_{j}$ for all $j<i$ and $\alpha_{i}<\beta_{i}$. Clearly, the inequalities $\alpha \leq \beta$ and $\beta \leq \alpha$ are equivalent to the equality $\alpha=\beta$. The partially ordered set $\left(\mathbb{Z}^{n}, \leq\right)$ is a linearly 
ordered set (for all distinct elements $\alpha, \beta \in \mathbb{Z}^{n}$ either $\alpha>\beta$ or $\alpha<\beta$ ) and $\alpha<\beta$ implies that $\alpha+\gamma<\beta+\gamma$ for all $\gamma \in \mathbb{Z}^{n}$. Every nonzero element $b=\sum_{\alpha \in \mathbb{Z}^{n}} b_{\alpha}$ of $A$ (where $b_{\alpha} \in A_{\alpha}$ ) can be written as

$$
b=b_{\alpha}+\cdots
$$

where $\alpha$ is the maximal element of $\operatorname{supp}(b)$ and the three dots denote smaller terms (i.e. the sum $\sum_{\beta<\alpha} b_{\beta}$ ). The term $b_{\alpha}=\lambda_{\alpha} v_{\alpha}$ is called the leading term of $b$, denoted lt $(b)$, and the element $\lambda_{\alpha} \in D$ is called the leading coefficient of $b$, denoted lc $(b)$. Since the algebra $A$ is a $\mathbb{Z}^{n}$-graded Poisson algebra, for all nonzero elements $b, c \in A$,

$$
\operatorname{lt}(b c)=\operatorname{lt}(b) \operatorname{lt}(c)
$$

provided $\operatorname{lc}(b) \operatorname{lc}(c) \neq 0$, and

$$
\operatorname{lt}(\{b, c\})=\{\operatorname{lt}(b), \operatorname{lt}(c)\}
$$

provided $\{\operatorname{lt}(b), \operatorname{lt}(c)\} \neq 0$.

Up to isomorphism in (8) (i.e. interchanging some $X_{i}$ and $Y_{i}$, if necessary), we can assume that the ideal $I$ contains a nonzero element $u=\lambda_{\alpha} X^{\alpha}+\cdots$ where $\alpha_{1} \geq 0, \ldots, \alpha_{n} \geq 0$. Then, the set of leading coefficients

$$
\mathfrak{a}=\left\{\lambda_{\alpha} \mid u=\lambda_{\alpha} X^{\alpha}+\cdots \in I, \text { all } \alpha_{i} \geq 0\right\}
$$

of elements of $I$ is a $\partial$-invariant ideal of the ring $D$ since

$$
\begin{aligned}
d_{1} u d_{2} & =d_{1} \lambda_{\alpha} d_{2} X^{\alpha}+\cdots & & \text { if } d_{1} \lambda_{\alpha} d_{2} \neq 0 \quad\left(d_{1}, d_{2} \in D\right), \\
u X^{\beta} & =\lambda_{\alpha} X^{\alpha+\beta}+\cdots, & & \\
\left\{u, X_{i}\right\} & =\partial_{i}\left(\lambda_{\alpha}\right) X^{\alpha+e_{i}}+\cdots & & \text { if } \partial_{i}\left(\lambda_{\alpha}\right) \neq 0 .
\end{aligned}
$$

Therefore, by condition 1 , there exists an element $u=X^{\alpha}+\cdots \in I$ (i.e. $\lambda_{\alpha}=1$ ). Then, using the equalities

$$
Y_{i} X_{i}^{\alpha}=a_{i} X^{\alpha-e_{i}} \text { and }\left\{Y_{i}, X^{\alpha}\right\}=\alpha_{i} \partial_{i}\left(a_{i}\right) X^{\alpha_{i}-e_{i}} \text {, }
$$

condition 2 and the fact that $\operatorname{char}(K)=0$ (condition 3), we can assume that $u=$ $1+\cdots \in I$, i.e. $u=1+\sum_{\alpha<0} u_{\alpha}$. For a finite set $S$, we denote by $|S|$ the number of its elements. Let

$$
m=\min \{|\operatorname{supp}(u)| \mid u=1+\cdots \in I\}
$$

We can assume that $|\operatorname{supp}(u)|=m$. The Poisson algebra $A$ is a $\mathbb{Z}^{n}$-graded Poisson algebra. Hence, by the choice of $m$, for all elements $d \in D$ and $i=1, \ldots, n$ :

$$
0=\{d, u\}=\sum_{\alpha<0}\left\{d, u_{\alpha}\right\}, \quad \text { i.e. }\left\{d, u_{\alpha}\right\}=0,
$$




$$
\begin{aligned}
& 0=\left\{X_{i}, u\right\}=\sum_{\alpha<0}\left\{X_{i}, u_{\alpha}\right\}, \quad \text { i.e. }\left\{X_{i}, u_{\alpha}\right\}=0, \\
& 0=\left\{Y_{i}, u\right\}=\sum_{\alpha<0}\left\{Y_{i}, u_{\alpha}\right\}, \quad \text { i.e. }\left\{Y_{i}, u_{\alpha}\right\}=0,
\end{aligned}
$$

i.e. all $u_{\alpha} \in \mathrm{PZ}(A)$, and so $0 \neq u \in \mathrm{PZ}(A)$, as required.

Corollary 3.4 Let $A=D[X, Y ; a$, $\partial\}$ be a GWPA of rank $n$. Suppose that the conditions 1 and 2 of Theorem 1.1 hold. Then, every nonzero Poisson ideal of $A$ intersects $\mathrm{PZ}(A)$ nontrivially.

Proof The corollary is precisely the Claim in the proof of Theorem 1.1.

Corollary 3.5 Let $D=K\left[H_{1}, \ldots, H_{n}\right]$ be a Poisson polynomial algebra with trivial Poisson bracket, $a=\left(a_{1}, \ldots, a_{n}\right)$ where $a_{i} \in K\left[H_{i}\right]$ and $\partial=\left(b_{1} \partial_{H_{1}}, \ldots, b_{n} \partial_{H_{n}}\right)$ where $b_{i} \in K\left[H_{i}\right]$. Then, the GWPA $A=D[X, Y ; a, \partial\}$ of rank $n$ is a simple Poisson algebra iff char $(K)=0, b_{1}, \ldots, b_{n} \in K^{*}:=K \backslash\{0\}$ and $K\left[H_{i}\right] a_{i}+K\left[H_{i}\right] \frac{d a_{i}}{d H_{i}}=$ $K\left[H_{i}\right]$ for $i=1, \ldots, n$.

Proof The corollary follows from Theorem 1.1. In more detail, condition 2 of Theorem 1.1 is equivalent to the conditions $K\left[H_{i}\right] a_{i}+K\left[H_{i}\right] \frac{d a_{i}}{d H_{1}}=K\left[H_{i}\right]$ for $i=1, \ldots, n$ (since $a_{i} \in K\left[H_{i}\right]$ ). Condition 1 of Theorem 1.1 is equivalent to the condition $\operatorname{char}(K)=0$ and $b_{1}, \ldots, b_{n} \in K^{*}:=K \backslash\{0\}$ (since $b_{i} D$ is a $\partial$-invariant ideal of $D$ ). If conditions 1 and 2 hold, then condition 3 of Theorem 1.1 holds automatically since $D^{\partial}=K=\mathrm{PZ}(D)$ (then $D_{\alpha}=0$ for all $\left.\alpha \in \mathbb{Z}^{n} \backslash\{0\}\right)$.

By Corollary 3.5, the classical Poisson polynomial algebra

$$
P_{2 n} \simeq K\left[H_{1}, \ldots, H_{n}\right]\left[X, Y ;\left(H_{1}, \ldots, H_{n}\right),\left(\partial_{H_{1}}, \ldots, \partial_{H_{n}}\right)\right\}
$$

is a simple Poisson algebra.

Open Access This article is distributed under the terms of the Creative Commons Attribution 4.0 International License (http://creativecommons.org/licenses/by/4.0/), which permits unrestricted use, distribution, and reproduction in any medium, provided you give appropriate credit to the original author(s) and the source, provide a link to the Creative Commons license, and indicate if changes were made.

\section{References}

1. Bavula, V.V.: Finite-dimensionality of $\operatorname{Ext}^{n}$ and $\operatorname{Tor}_{n}$ of simple modules over a class of algebras. Funct. Anal. Appl. 25(3), 229-230 (1991)

2. Bavula, V.V.: The simple $D[X, Y ; \sigma, a]$-modules. Ukrainian Math. J. 44(12), 1500-1511 (1992)

3. Bavula, V.V.: Generalized Weyl algebras and their representations. St. Petersburg Math. J. 4(1), 71-92 (1993)

4. Cho, E.-H., Sei-Qwon, O.: Semiclassical limits of ore extensions and a Poisson generalized Weyl algebra. Lett. Math. Phys. 106, 997-1009 (2016)

5. Dixmier, J.: Sur les algèbres de Weyl. Bull. Soc. Math. France 96, 209-242 (1968)

Publisher's Note Springer Nature remains neutral with regard to jurisdictional claims in published maps and institutional affiliations. 